\title{
The Sanford Underground Research Facility at Homestake
}

\author{
J. Heise \\ Sanford Underground Research Facility, 630 East Summit Street, Lead, SD 57754
}

\begin{abstract}
The former Homestake gold mine in Lead, South Dakota is being transformed into a dedicated laboratory to pursue underground research in rare-process physics, as well as offering research opportunities in other disciplines such as biology, geology and engineering. A key component of the Sanford Underground Research Facility (SURF) is the Davis Campus, which is in operation at the 4850-foot level (4300 m.w.e) and currently hosts three projects: the LUX dark matter experiment, the MAJORANA DEMONSTRATOR neutrinoless double-beta decay experiment and the CUBED low-background counter. Plans for possible future experiments at SURF are well underway and include long baseline neutrino oscillation experiments, future dark matter experiments as well as nuclear astrophysics accelerators. Facility upgrades to accommodate some of these future projects have already started. SURF is a dedicated facility with significant expansion capability.
\end{abstract}

Keywords: Underground laboratory, dark matter, neutrino, double-beta, nuclear astrophysics PACS: $14.60 . \mathrm{Pq}, 23.40 .-\mathrm{s}, 26.00,95.35 .+\mathrm{d}$

\section{INTRODUCTION}

Many disciplines benefit from access to an underground facility dedicated to scientific research, including physics, biology, geology, engineering, and a well-established science program is currently underway at the Sanford Underground Research Facility (SURF). The unique underground environment at SURF allows researchers to explore a host of important questions regarding the origin of life and its diversity, mechanisms associated with earthquakes and a number of engineering topics. A deep underground laboratory is also where some of the most fundamental topics in physics can also be investigated, including the nature of dark matter, the properties of neutrinos and the synthesis of atomic elements within stars.

SURF is being developed in the former Homestake gold mine, in Lead, South Dakota [1, 2]. Barrick Gold Corporation donated the site to the State of South Dakota in 2006, following over 125 years of mining [3], during which time over $600 \mathrm{~km}$ of tunnels and shafts were created in the facility, extending from the surface to over 8,000 feet below ground. The Laboratory property comprises 186 acres on the surface and 7,700 acres underground, and the Surface Campus includes approximately 253,000 gross square feet of existing structures. The South Dakota Science and Technology Authority (SDSTA) operates and maintains the Sanford Laboratory at the Homestake site in Lead, South Dakota with management oversight by Lawrence Berkeley National Laboratory (LBNL).

In 2006, South Dakota philanthropist, T. Denny Sanford, gifted \$70M to convert the former mine into a research laboratory and develop a science education facility. With these funds and a strong commitment from the State of South Dakota (appropriations of $\$ 42.2 \mathrm{M}$ to date), safe access to the underground has been reestablished.

The initial concepts for SURF were developed with the support of the US National Science Foundation (NSF) as the primary site for the NSF's Deep Underground Science and Engineering Laboratory (DUSEL) [4]. With the National Science Board's decision to halt development of a NSF-funded underground laboratory, the US Department of Energy (DOE) now supports the majority of the operation of the facility. Support for experiments at SURF comes from both the NSF and DOE as well as other agencies such as the USGS and NASA. Elements of the Homestake DUSEL Preliminary Design Report [5] continue to be useful as the feasibility for portions of the original plan are investigated.

\section{FACILITY OPERATIONS INFRASTRUCTURE}

Maintenance and operation of key elements of facility infrastructure enables safe access underground. Transportation of personnel and materials underground is accomplished using the two primary shafts, the Ross Shaft and the Yates Shaft. Pairs of hoists near both the Ross and Yates shafts move personnel and rock conveyances through the respective shafts. Pumping stations in the Ross Shaft allow ground water to be pumped to surface. Underground ventilation is 


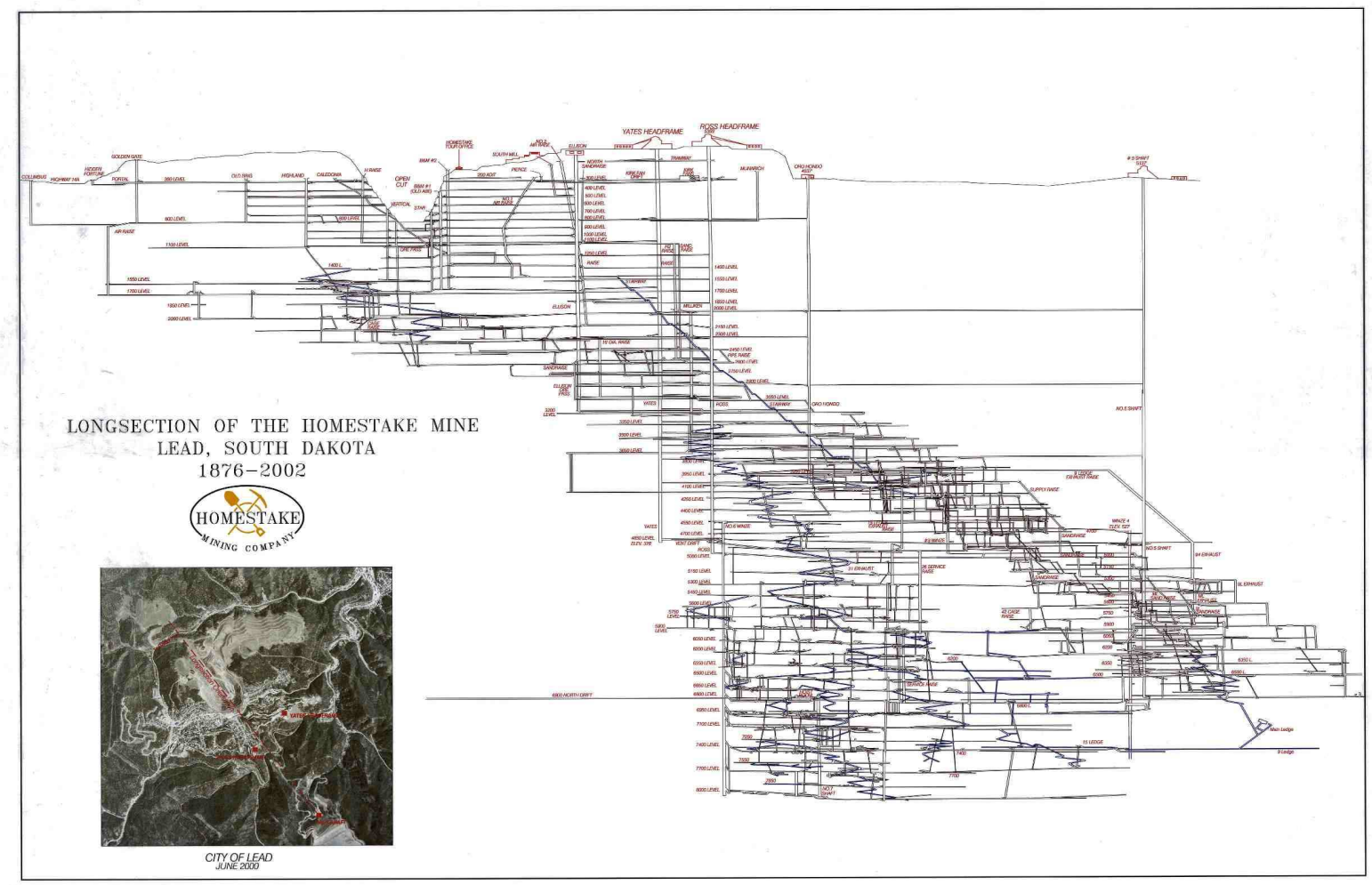

FIGURE 1. The long section of the former Homestake Gold Mine. This figure illustrates the 60 underground levels extending to greater than 8,000 feet below ground. The location of cross section is indicated in the inset along a NW to SE plane. The projection extends for $5.2 \mathrm{~km}$ along this plane.

provided by the Oro Hondo fan as well as the fan at \#5 Shaft, which bring fresh air underground via the Ross and Yates Shafts.

A key feature of the Sanford Laboratory is the capacity for redundancy. Redundant power, fiber optics cable and ventilation are brought underground via the Ross and Yates Shafts. As well, the two shafts as well as numerous ramp systems provide multiple options for emergency egress.

Initial rehabilitation of the surface and underground infrastructure focused on the Ross shaft that provides access to the majority of underground utilities, including the pumping system used to remove water from the mine. Contractor work in the Ross shaft started July 2008 and was completed by October 2008, after which SDSTA personnel continued with maintenance and renovations, including the removal of unused legacy piping $(12 \mathrm{~km})$ and power/communication cables (2 km). Started in November 2008, the Yates shaft renovation was completed in May 2012 with the installation of a new personnel conveyance and emergency braking system. The Ross Shaft provided primary underground access from the start of re-entry in 2007 until the Yates shaft was ready in May 2012. Personnel and materials are transported underground mainly using the Yates cage, which has dimensions $1.4 \mathrm{~m}$ wide $\times 2.7 \mathrm{~m}$ tall $\times 3.8 \mathrm{~m}$ long and has a maximum load capacity of $10,000 \mathrm{lbs}(4,536 \mathrm{~kg})$. The Yates cage schedule accommodates three shifts per day for science personnel, providing 24-hour access as needed.

The average ground water inflow into the underground workings is approximately $730 \mathrm{gpm}$. After pumping ceased in June 2003 [6, 7], the mine filled with water until a high-water mark of 1381 meters ( 4530 feet) below surface was reached in August 2008. Sustained pumping resumed in June 2008, dropping the water level below the 4850 Level by May 2009. Since April 2012 the water level is being maintained around the 6000-foot level below surface. While the potential for accommodating deeper access exists, without a funded mandate to develop laboratory space below the 4850-foot level, there is benefit in terms of cost and safety for maintaining the water level around the current level.

A deep-well pump was installed July 2010 and is currently located about 6424 feet below surface in \#6 Winze, which extends from the 4850 Level to the deepest areas of the facility. Permanent stations employing 700 -horsepower pumps are located on the 1250, 2450, 3650 and 5000 Levels in the Ross shaft. Water received at the surface Waste Water 
Treatment Plant (WWTP) is combined with Homestake-Barrick water and treated to remove iron that has leached from the mine workings and trace amounts of ammonia. The discharge capacity from the WWTP is roughly $2000 \mathrm{gpm}$ using biological and sand-filter technologies.

Single-mode fiber optics cable is deployed throughout the facility and current network hardware provides intercampus communication at 100-1000 Mbps. Redundant connections exist to the outside world, including commodity internet ("Internet 1") at $1 \mathrm{Gbps}$ and research internet ("Internet 2" via the state Research, Education and Economic Development (REED) network) at $1 \mathrm{Gbps}$, which can be expanded to $100 \mathrm{Gbps}$ with appropriate hardware upgrades. Redundant fiber connections connect the surface to the 4850L via the Ross and Yates Shafts, and work in underway to ensure that the core network equipment is protected by uninterruptible power supplies and in most cases generator power.

In order to provide increased capacity to support the construction and operation of future experiments, state and private funds have been allocated to perform extensive renovations in the Ross Shaft. Refurbishment of the Ross Shaft began in August 2012, and over 1100 feet of new steel and associated ground support has been installed as of December 2013. A total of 5159 feet of new steel sets will be installed as part of the Ross shaft upgrade project, which is expected to be completed by mid-2017. Once the Ross shaft is completed, a planned renovation of the Yates Shaft will commence.

\section{SCIENCE FACILITIES}

In addition to the considerable underground extent, several dedicated facilities exist at SURF to support science activities. On the surface, a number of spaces are being used for storage (including drill core) and limited construction activities. Three main physics laboratory spaces are available: the Surface Laboratory and two campuses on the 4850 Level - the Ross Campus and the Davis Campus.

\section{Surface Facilities}

Key surface facilities at SURF support science activities at both the Yates and Ross surface campuses, including administrative activities, education and public outreach functions, the Waste Water Treatment Plant (WWTP) and a warehouse for shipping/receiving. Figure 2 shows the extent of the surface property.

While there are many possibilities of surface buildings that could be renovated to directly support science activities, two facilities currently function in that capacity: the Core Archive and the Surface Laboratory.

\section{Core Archive}

Donated by Homestake-Barrick, SURF is the steward of 39,760 boxes of drill core, which corresponds to a total length of $91 \mathrm{~km}$. Homestake core holes extend to 10,800 feet below surface. An additional 5,400 feet of core were added to the collection as part of the geotechnical investigations on the 4850L for DUSEL.

The SD Geological Survey has assisted with the development of an online database that so far includes 58,000+ entries, representing 1,740 drill holes.

\section{Surface Laboratory}

Renovations were undertaken in 2009 in order to transform a former warehouse into a laboratory. Construction was completed in early 2010, resulting in approximately $190 \mathrm{~m}^{2}$ of lab space in the top-most level of a four-storey building as shown in Figure 3. The facility includes a cleanroom $(5.6 \mathrm{~m} \times 6.6 \mathrm{~m}$ with a $2.7 \mathrm{~m}$ ceiling height $)$ and corresponding dedicated air handling and filtration system as well as a tank that can be used as a water shield ( $2.8 \mathrm{~m}$ diameter $\times$ $4 \mathrm{~m}$ high). The tank is installed in a recessed shaft in the center of the laboratory space. The laboratory was initially designed to meet the needs of the LUX experiment, but is now used to support multiple research groups. 


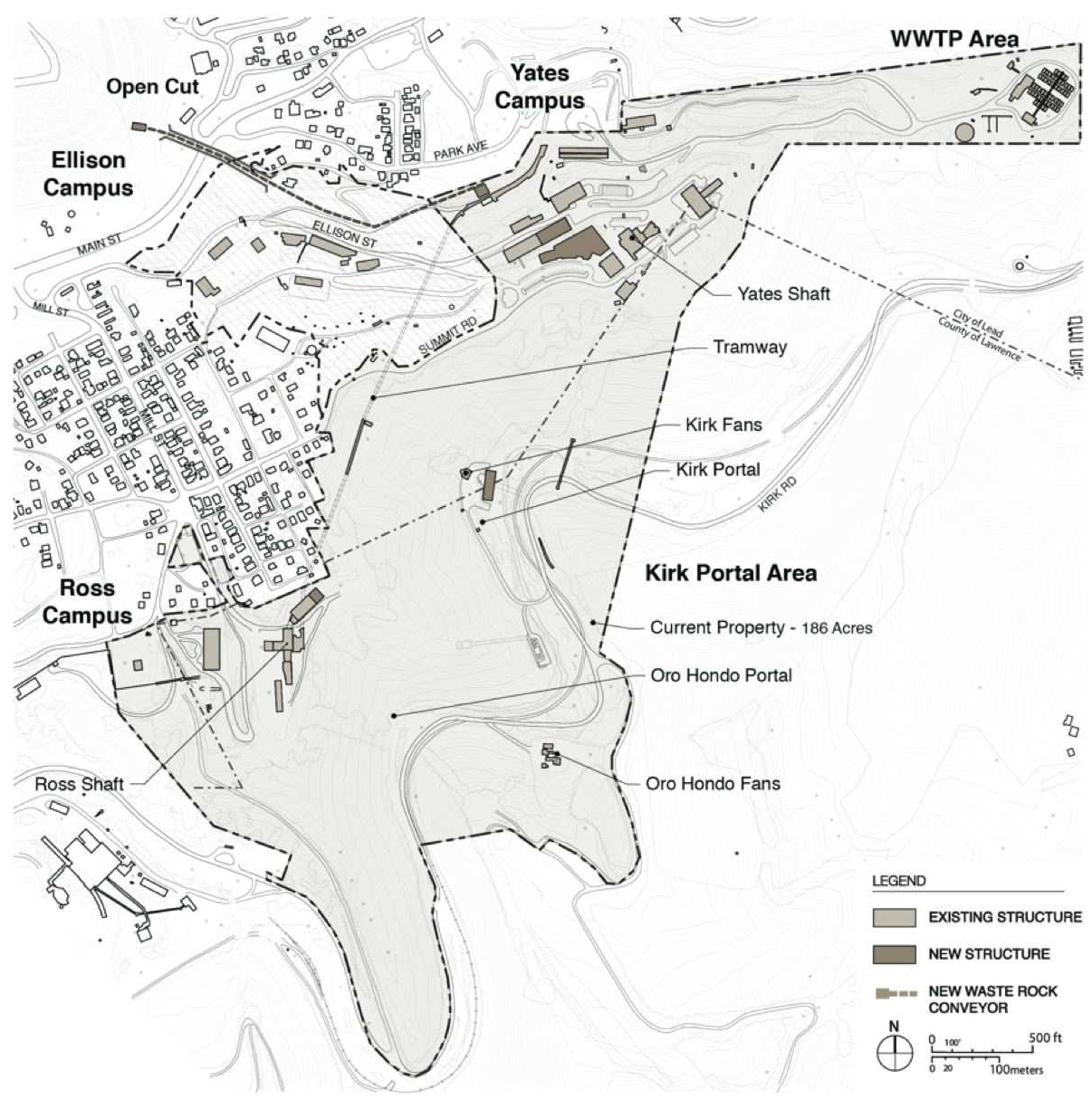

FIGURE 2. A plan view of the surface campus at SURF, which comprises 186 acres.

\section{Underground Facilities}

A number of levels required to support facility operations infrastructure can also accommodate research activities. However, the main infrastructure for the support of science activities has been developed on the 4850L with formal campuses located near both the Ross and Yates shafts.

\section{L Ross Campus}

The 4850L Ross Campus includes the Ross Shaft and \#6 Winze and encompasses a set of four existing excavations that were used as maintenance shops during mining activities. These former shops afford an economical means to implement experiments or other equipment in a timely manner. The layout is shown in Figure 4, including the location of the current electrical substation and generators.

Two of the shop areas are currently in use. The SW area has been converted into a safety Refuge Chamber that can accommodate 72 people for up to 96 hours. It includes compressed air, $\mathrm{CO}_{2}$ scrubbers, air conditioners, rations and communications. The NW area is currently being used by the MAJORANA collaboration to electroform copper as shown in Figure 5.

The NE shop will house the CASPAR accelerator and a cleanroom is proposed for the SE area to host a suite of low-background counters and possibly support other research activities, too. 


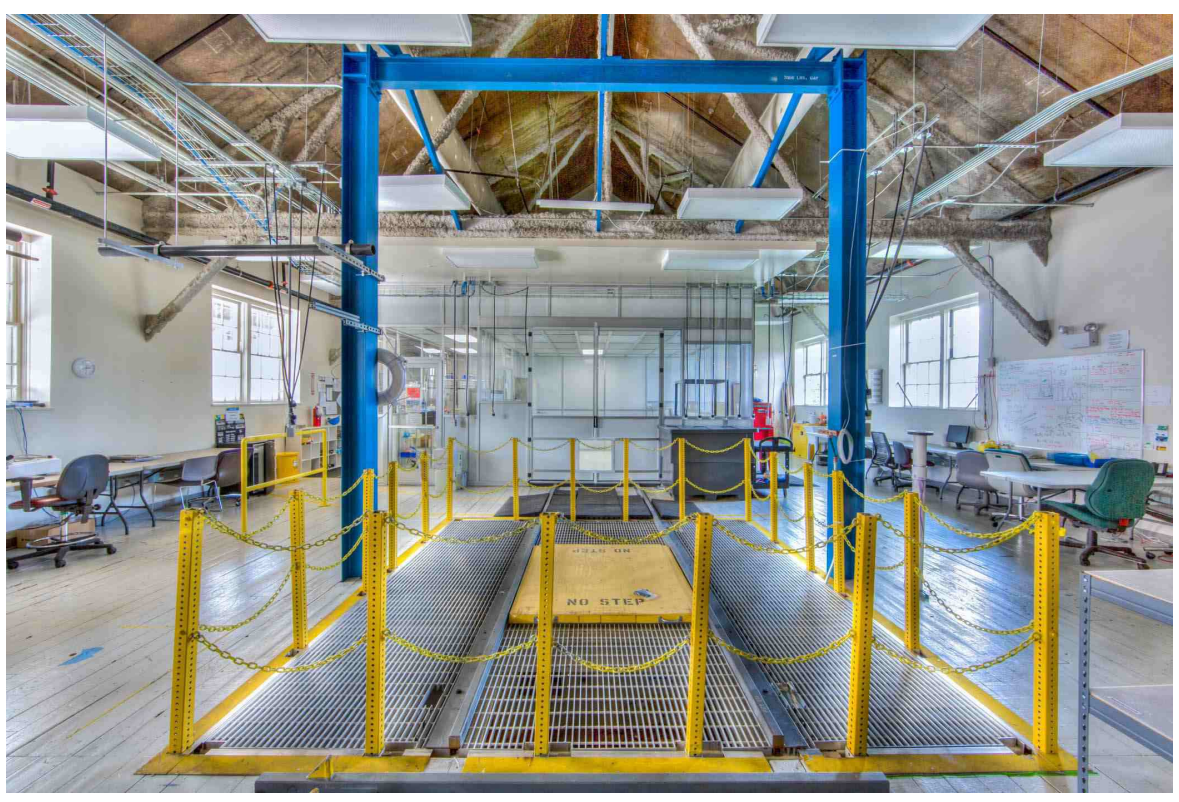

FIGURE 3. Surface Laboratory. The recessed area with the tank is under the grating in the foreground and the cleanroom is located in the background. A hoist can be installed on the blue I-beam structure.

TABLE 1. Footprint areas and volumes for the 4850L Ross Campus spaces. The volume for the NE area is derived from laser-scan data.

\begin{tabular}{lcc}
\hline Ross Campus Location & $\begin{array}{c}\text { Area } \\
\left(\mathbf{m}^{2}\right)\end{array}$ & $\begin{array}{c}\text { Volume } \\
\left(\mathbf{m}^{3}\right)\end{array}$ \\
\hline NW (MAJORANA Electroforming) & 184 & 504 \\
NE & 297 & 707 \\
SE & 137 & 376 \\
SW (Refuge Chamber) & 121 & 332 \\
\hline
\end{tabular}

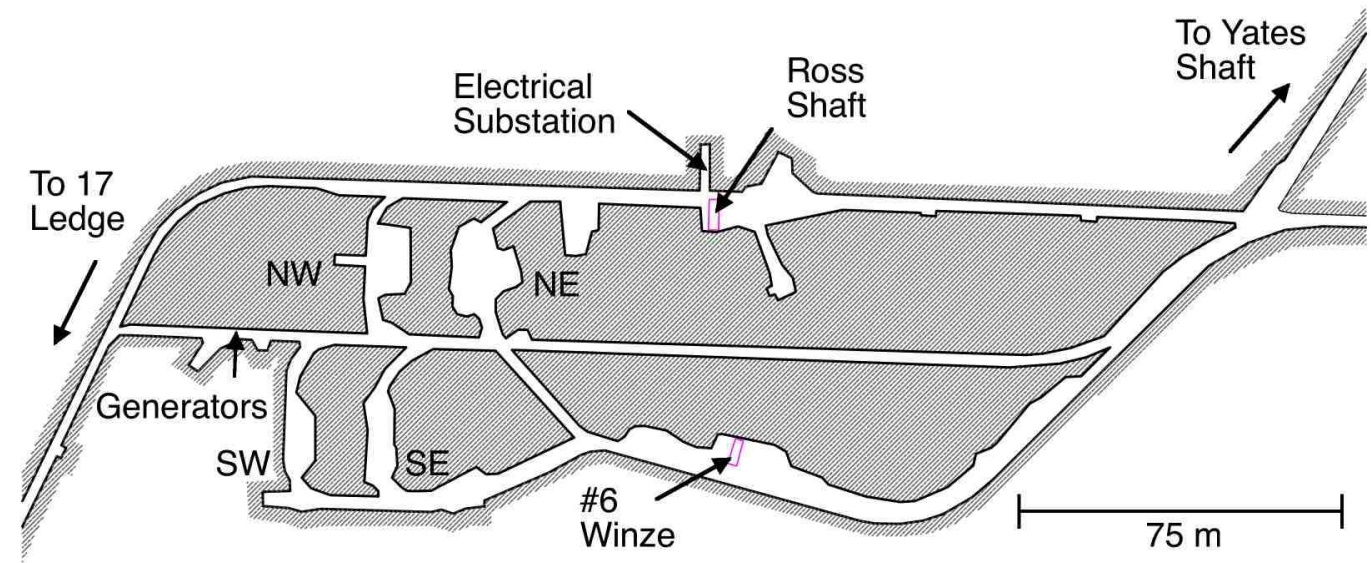

FIGURE 4. 4850L Ross Campus. Four existing excavations are labeled: NW, NE, SE, SW. 


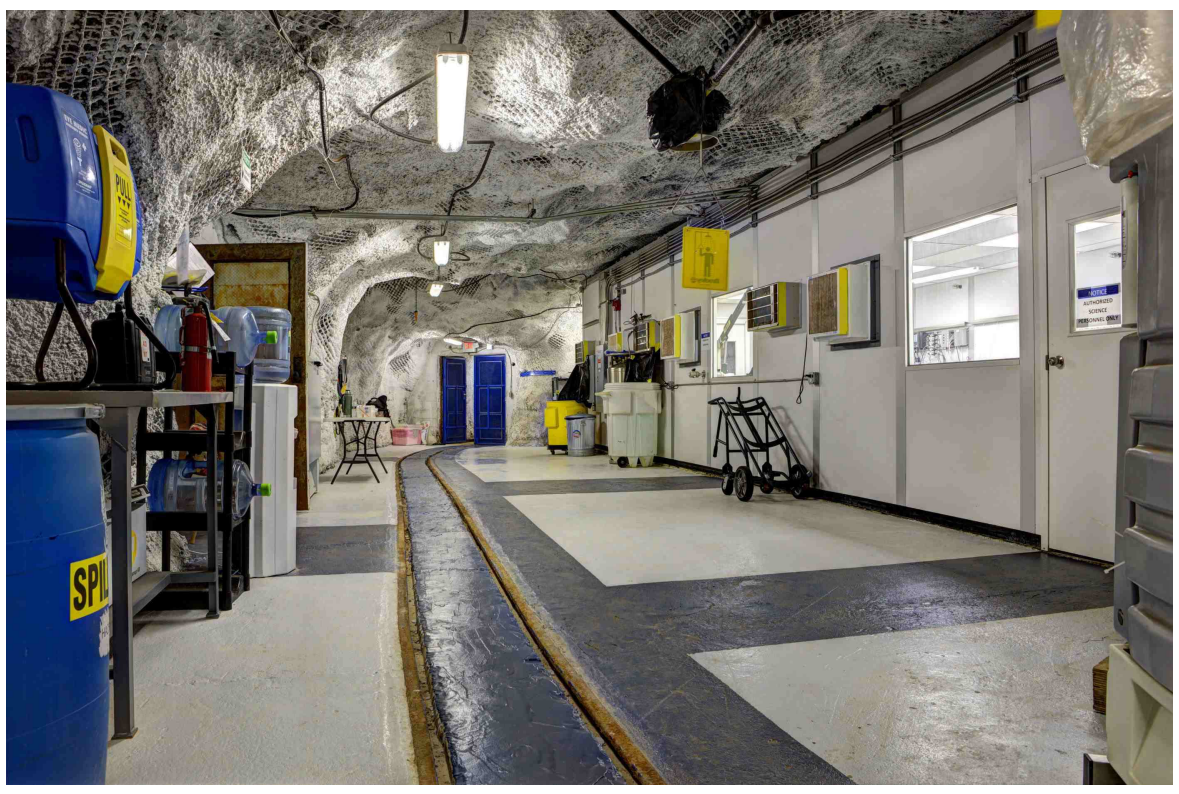

FIGURE 5. The MAJORANA electroforming laboratory at the 4850L Ross Campus. The cleanroom is located on the right-hand side.

\section{L Davis Campus}

A state-of-the-art laboratory complex called the Davis Campus has been constructed at the 4850L near the Yates Shaft. The Davis Campus represents a \$15.2M South Dakota commitment using state and private funds. New excavation for the Davis Campus took place during September 2009 - January 2011, during which time 16,632 tonnes (18,334 tons) of rock was removed. Rather than being transported to the surface, areas were identified on the 5000L (via the 4850L) for rock storage. Shotcrete was applied in both the Davis Cavern (average thickness $12.7 \mathrm{~cm}$ ) and the Transition space (average thickness $8.9 \mathrm{~cm}$ ). Laboratory outfitting began in June 2011 and was substantially complete in May 2012; pictures are shown in Figure 7. A final occupancy certificate was issued in July 2012, which specifies the maximum number of occupants to be 62 persons.

The two main experiments areas are the MAJORANA Lab/Transition space $\left(43 \mathrm{~m} \mathrm{~L} \times 16 \mathrm{~m} \mathrm{~W} \times 4 \mathrm{~m} \mathrm{H}^{2} 3,648 \mathrm{~m}^{3}\right)$ and the Davis Cavern $\left(17 \mathrm{~m} \mathrm{~L} \times 10 \mathrm{~m} \mathrm{~W} \times 12 \mathrm{~m} \mathrm{H} ; 2,089 \mathrm{~m}^{3}\right)$, as shown in Figure 6. Quoted dimensions are average values based on post-shotcrete laser-scan data. The total Davis Campus footprint consists of $927 \mathrm{~m}^{2}$ of laboratory space and $2,732 \mathrm{~m}^{2}$ total space.

An array of extensometers is installed to monitor ground motion and convergence at the Davis Campus - data collected so far indicate nothing unexpected. Geotechnical interpretations were performed using drill core and other rock mechanics studies [8].

Services provided within the Davis Campus include fire sprinklers and alarms throughout the area, potable and non-potable (industrial) water, lighting, emergency lighting, ventilation and air conditioning. A building management system provides controls throughout the Campus. Cooling is provided with two redundant 50-ton (633 MJ) chillers supplying chilled water to three air handling units that provide ventilation to separate Campus spaces. Chilled water is also available for experiments to connect equipment directly. A dedicated $1500 \mathrm{kVA}$ substation provides sufficient capacity for the experiment and facility needs, with margin for future expansion. Emergency power for lighting is provided with batteries in the lighting system to provide immediate light, while a standby diesel generator near the campus provides up to 24 hours of power to all safety systems in the campus. This includes water pumps in the nearby Yates shaft to prevent water from rising into the campus spaces.

While space is at a premium at the Davis Campus, two cutouts exist outside the clean space offering footprints of $33-50 \mathrm{~m}^{2}$, with an average ceiling height of $3.7 \mathrm{~m}$. These spaces could be made available to groups with modest equipment needs. 


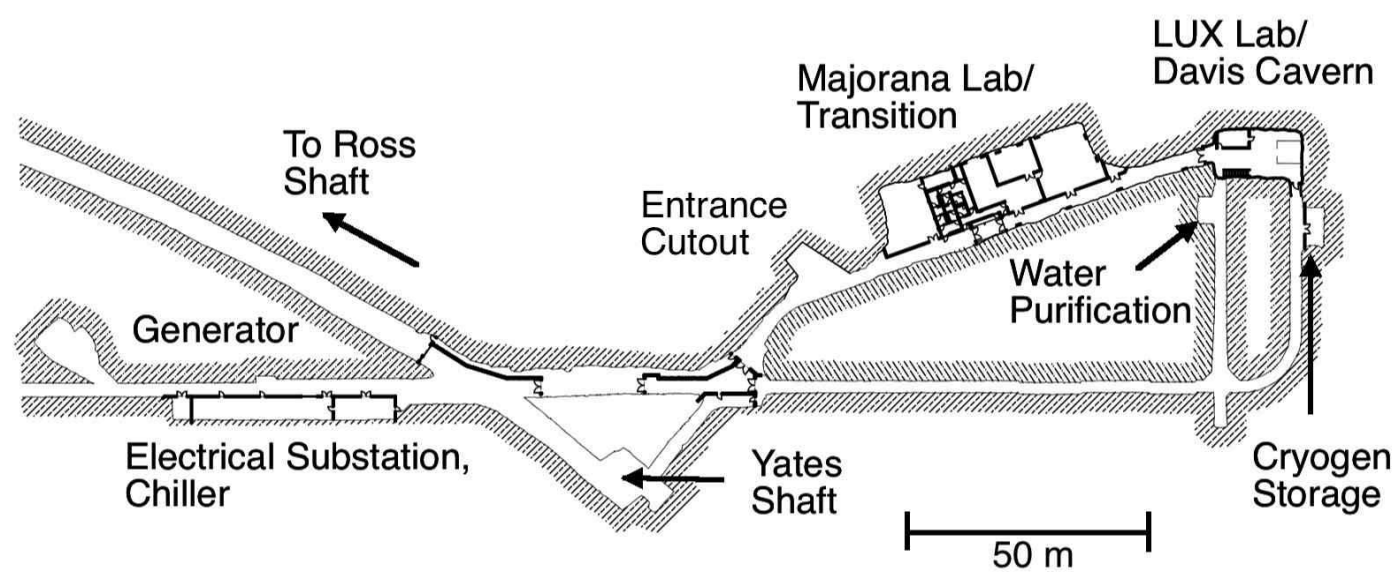

FIGURE 6. 4850L Davis Campus showing the two main laboratory spaces and the proximity to the Yates Shaft.

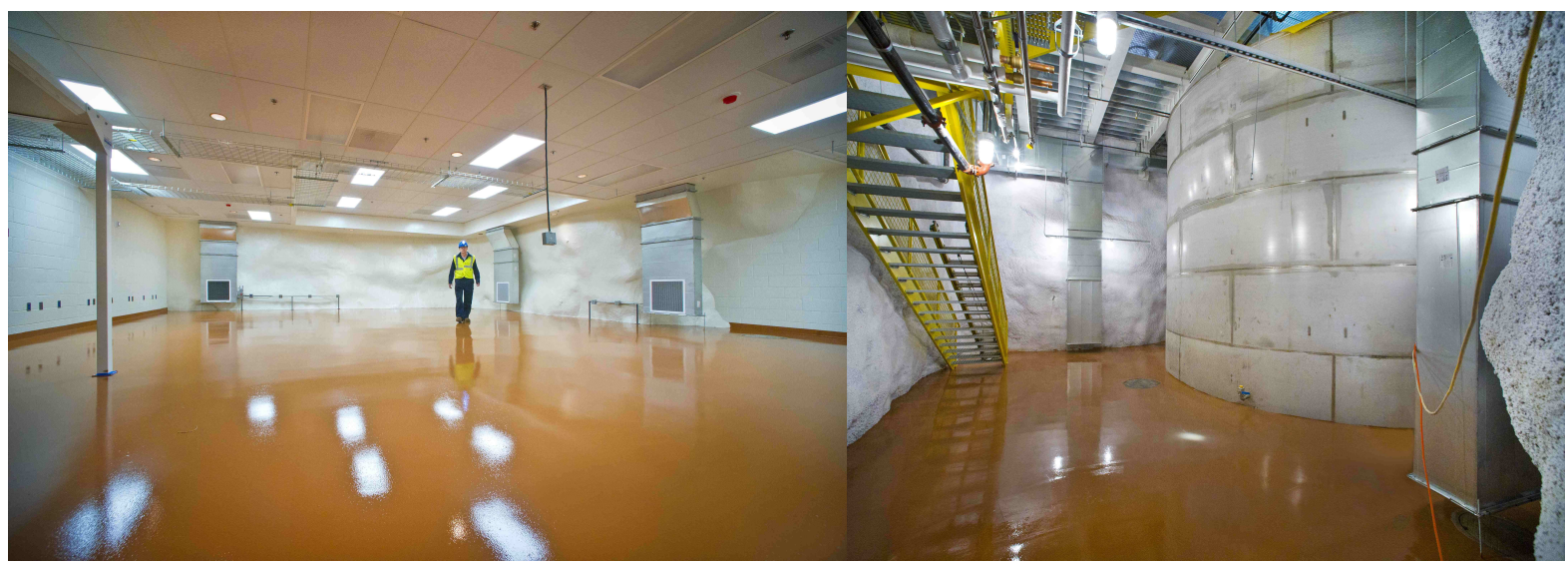

FIGURE 7. Pictures of the Davis Campus in May 2012 after outfitting had finished: (upper) MAJORANA Detector Room, (lower) water shielding tank in Lower Davis for the LUX detector. A considerable amount of equipment has since been installed by both collaborations.

\section{Rock Overburden}

When considering the muon flux at key laboratory spaces on the 4850L, six geological formations are important to consider: Grizzly, Flagrock, Northwestern, Ellison, Homestake and Poorman. The Yates Member (Unit) is the lowest stratigraphic unit of the Poorman Formation and is important for developments on the 4850L. In addition, tertiary Rhyolite dikes occur throughout the rock mass.

The surface topology varies significantly throughout the laboratory property as shown in Figure 8. Using average rock densities and preliminary geological model data in addition to recent topological surveys, an estimate of the rock overburden can be determined as shown in Table 2. Overburden uncertainties will be quantitatively evaluated in an upcoming publication. Geochemical composition data for various formations are also being compiled from sources such as the USGS [9] and others. 


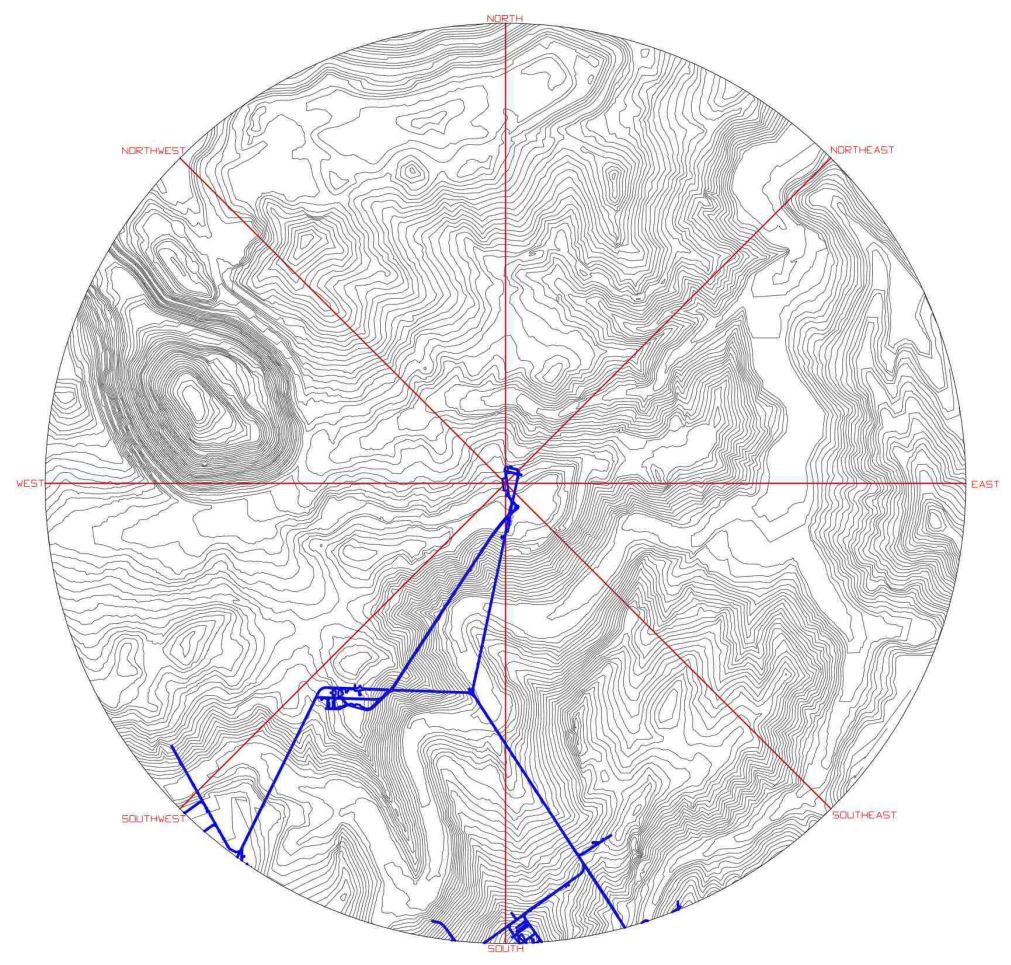

FIGURE 8. Topological contours overlaid on top of an outline of the 4850L (shown in blue). Various cardinal coordinates are indicated with red lines. The Open Cut is a prominent feature on the surface toward the west. Contour lines indicate 20-foot elevation changes.

TABLE 2. Rock overburden estimates for different underground laboratory locations at SURF, reflecting considerable variations in surface topology as well as geology. Uncertainties on the raw overburden values are on the order of a few percent.

\begin{tabular}{lcc}
\hline 4850L Laboratory & \multicolumn{2}{c}{ Rock Overburden } \\
Location & (m) & (m.w.e) \\
\hline Davis Campus - LUX & 1464 & 4200 \\
Davis Campus - MJD & 1478 & 4300 \\
Davis Campus - Entrance Cutout & 1561 & 4400 \\
Ross Campus - MJD Electroforming & 1503 & 4300 \\
Ross Campus - LBNE & 1386 & 3900 \\
\hline
\end{tabular}

\section{Cleanliness}

Cleanliness at the Davis Campus is maintained through a combination of transition zones and protocols as well as dedicated cleaning staff. When supplies and equipment are brought from the dirty space into the clean space, items either need to be suitably enclosed or cleaned in the facility entrance Cart Wash. Personnel typically enter though a series of rooms where outer coveralls and dirty-side gear is removed in favor of corresponding clean items. Laundry services are also available in the clean space.

When the laboratory is occupied, particle counts in all common areas are below 10,000/ $\mathrm{ft}^{3}$. The MAJORANA collaboration follows additional gowning protocols to achieve ultra-low cleanliness levels in their laboratory spaces. A summary of cleanliness states throughout various areas is presented in Table 3 . The facility design includes two sets of showers, but so far they have not be necessary. 
TABLE 3. Median particle count levels in various laboratory locations during occupied periods.

\begin{tabular}{lc}
\hline Davis Campus Location & $\begin{array}{c}\text { Particle Count } \\
\mathbf{( 0 . 5} \boldsymbol{\mu \mathbf { m } / \mathbf { f t } ^ { 3 } )}\end{array}$ \\
\hline Transition, Laundry Area & $1290-8200$ \\
Transition, Common Corridor & 3160 \\
Davis Cavern, Lower & 3400 \\
Davis Cavern, Lower Counting Room & 710 \\
Davis Cavern, Upper & 450 \\
Transition, MAJORANA Detector Room & $100-400$ \\
\hline
\end{tabular}

\section{Radioactivity and Radon}

SURF strives to provide the lowest possible radioactivity environment for experiments hosted within the facility. This commitment had been integrated into the site preparation process from the early days of the facility design, and carried over to the realization of the 4850L Davis Campus laboratories. These efforts include site and environmental characterization including rock radioactive measurements, use of low-radioactivity construction materials, and regular monitoring of environmental factors, including airborne radon.

The natural abundance of $\mathrm{U} / \mathrm{Th} / \mathrm{K}$ in Homestake rock is generally low, especially in certain geological formations such as the Yates Amphibolite, which have been measured to contain sub-ppm levels of U/Th. Samples from other formations such as the Rhyolite intrusions can be 30-40x higher as illustrated in Table 4. While not as low as the host Amphibolite rock, the lowest-activity construction materials for the Davis Campus were selected from a large number of samples $[10,11,12]$.

TABLE 4. Partial U/Th/K assay results for Homestake rock samples as well as key construction materials used at the Davis Campus [10, $11,12]$.

\begin{tabular}{lccc}
\hline & $\begin{array}{c}\text { Uranium } \\
(\mathbf{p p m})\end{array}$ & $\begin{array}{c}\text { Thorium } \\
\mathbf{( p p m )}\end{array}$ & $\begin{array}{c}\text { Potassium } \\
\mathbf{( \% )}\end{array}$ \\
\hline Amphibolite & 0.22 & 0.33 & 0.96 \\
Rhyolite Dike & 8.75 & 10.86 & 4.17 \\
Shotcrete - Low Activity & 1.52 & 2.17 & 0.55 \\
Shotcrete - Standard & 2.00 & 3.35 & 1.23 \\
Shotcrete - Finish Coat & 1.62 & 3.08 & 0.79 \\
Masonry Blocks & 2.16 & 3.20 & 1.23 \\
\hline
\end{tabular}

Long-term underground radon data have been collected since 2009 and at the Davis Campus since July 2012, shortly after outfitting was completed. Aside from the normal facility ventilation that brings fresh air via the Ross and Yates shafts, no extraordinary steps have been taken to mitigate underground radon levels. Some research groups employ a type of purging system to reduce radon locally for their equipment. As seen at other underground laboratories, a seasonal dependence is becoming apparent in the Davis Campus trends, with higher radon levels observed during the summer months May through September. The total average radon concentration for all periods at the Davis Campus is approximately $340 \mathrm{~Bq} / \mathrm{m}^{3}$ with a low baseline of $150 \mathrm{~Bq} / \mathrm{m}^{3}$ during the winter months.

Other efforts to characterize physics backgrounds (eg., muons, neutrons, gamma rays) in various underground areas were carried out by research groups over several years $[13,14,15]$. Results from recent neutron flux measurements collected on the 4850L are expected in an upcoming publication.

\section{CURRENT SCIENCE PROGRAM}

Science efforts that started in 2007 during re-entry into the facility have grown steadily over the years. Building on the legacy of the Ray Davis chlorine solar-neutrino experiment [16] that began in 1965 at the Homestake Mine, more than a dozen research groups have established underground research programs at SURF. Measurements have been made 
and samples collected from elevations ranging from surface to the 5000-foot level, investigating topics in physics, geology, biology and engineering. Research programs that are considered to be currently active are listed in Table 5 as well their respective locations. Three main physics efforts are underway at SURF.

\section{Large Underground Xenon (LUX)}

The LUX experiment is conducting a direct search for weakly interacting massive particles (WIMPs) using $350 \mathrm{~kg}$ of purified xenon inside an ultra-pure titanium cryostat that is immersed in a water tank with 272,550 liters (72,000 gallons) of purified water. Following collisions in the liquid xenon volume, a strong electric field moves ionization electrons into the gas space at the top of the detector, and a total of 122 PMTs collect scintillation light from interactions in both xenon volumes.

Members of the LUX collaboration have been active onsite at SURF since December 2009, when preparations began for detector assembly at the Surface Laboratory. After completing detector assembly and operational testing on surface, LUX began their transition underground to the Davis Campus in May 2012. With strong support from SURF personnel, the fully-assembled LUX detector was successfully transported underground from the Surface Laboratory in the Yates cage and into the Davis Cavern - stringent limits on acceleration and tilt were not exceeded. The LUX space at the Davis Campus includes a cleanroom and a control room, which is separate from the main lab space. The SURF water purification system and a storage area for liquid nitrogen are located near the LUX equipment but outside the clean space.

The LUX collaboration recently published results from their first underground data run [17].

\section{Majorana Demonstrator (MJD)}

The MAJORANA collaboration is investigating neutrinoless double-beta decay using a detector called the DEMONSTRATOR consisting $40 \mathrm{~kg}$ of germanium in two ultra-pure copper cryostats and a compact lead shield. MAJORANA expects to use up to $30 \mathrm{~kg}$ of enriched ${ }^{76} \mathrm{Ge}$. The main technical goal of the DEMONSTRATOR is to confirm that the ambitious purity requirements for a tonne-scale detector are achievable [18]. The group also hopes to test a controversial claim for the detection of neutrinoless double-beta decay.

In order to satisfy the very low background requirements, the MAJORANA collaboration has set up a laboratory at the 4850L Ross Campus to produce the world's purest electroformed copper, which is then machined at a dedicated machine shop at the Davis Campus.

The MAJORANA collaboration began work onsite starting in November 2010 with deliveries of equipment (including the first germanium detectors) and then with preparations for the electroforming cleanroom. Production of electroformed copper began in July 2011 and about eight months later the group started to move equipment into the Davis Campus.

\section{Center for Ultra-low Background Experiments in the Dakotas (CUBED)}

CUBED is a South Dakota Governor's Research Center that is administered through the University of South Dakota and involves physics researchers and others from the majority of universities in the state. In addition to increasing the South Dakota academic involvement in SURF research, the center is maintaining a focus on areas of interest congruent with planned SURF experiments, such as crystal growth, low-background counting and isotope separation.

A low-background counting laboratory has been set up in a dedicated room in the Lower Davis Cavern. The system allows direct-gamma counting using a 1.2-kg high-purity germanium crystal, resulting in $0.01-0.1 \mathrm{ppb}$ sensitivities to U/Th. Initial operation has begun, and production counting is expected to start in early 2014 once the outer shielding is complete.

Another CUBED project that proposes to investigate isotopic separation and ultra-purification techniques is being considered for the Surface Laboratory. Funding is being sought to develop a laboratory to support underground crystal growth. 
TABLE 5. Current scientific research programs at the Sanford Laboratory. Locations in bold indicate current installations or the subject of current focus.

\begin{tabular}{|c|c|c|c|}
\hline Experiment & Description & Locations & References \\
\hline \multicolumn{4}{|l|}{ Physics } \\
\hline LUX & Dark matter using $\mathrm{Xe}$ & Surface, $4850 \mathrm{~L}$ & {$[17]$} \\
\hline MAJORANA DEMONSTRATOR & Neutrinoless double-beta decay using Ge & Surface, $4850 \mathrm{~L}$ & {$[18]$} \\
\hline $\begin{array}{l}\text { CUBED } \\
\text { (also Bkgd Characterization) }\end{array}$ & $\begin{array}{l}\text { Low-bkgd counter; also bkgds: } \\
\text { muon, neutron, gamma, radon }\end{array}$ & $\begin{array}{l}\text { Surface, 800L, } \\
\text { 2000L, 4550L, 4850L }\end{array}$ & [19], [13], [14], [15] \\
\hline DIANA & Neutron bkgds & $4100 \mathrm{~L}, 4850 \mathrm{~L}$ & * \\
\hline LBNE & Cleanliness tests & Surface, $4850 \mathrm{~L}$ & {$[20]$} \\
\hline $\begin{array}{l}\text { Deep Underground Gravity } \\
\text { Laboratory (DUGL) }\end{array}$ & $\begin{array}{l}\text { Seismic characterization for gravity-wave } \\
\text { research }\end{array}$ & $\begin{array}{l}\text { Surface, 300L, } \\
800 \mathrm{~L}, 2000 \mathrm{~L}, 4100 \mathrm{~L}\end{array}$ & [21], [22] \\
\hline \multicolumn{4}{|l|}{ Geology } \\
\hline $\begin{array}{l}\text { Geoscience Optical Extensometers } \\
\text { and Tiltmeters }\left(\mathrm{GEOX}^{\mathrm{TM}}\right)\end{array}$ & Optical fiber apps, tiltmeters & $\begin{array}{l}\text { 2000L, } 4100 \mathrm{~L}, \\
4850 \mathrm{~L}\end{array}$ & [23], [24], [25], [26], [27] \\
\hline USGS Hydrogravity & Local gravity for water tables, densities & Surface, $4100 \mathrm{~L}, 4850 \mathrm{~L}$ & {$[28]$} \\
\hline $\begin{array}{l}\text { Petrology, Ore Deposits, } \\
\text { Structure (PODS) }\end{array}$ & Core archive and logs, geologic mapping & Surface, $800 \mathrm{~L}$ & [29], [30] \\
\hline Transparent Earth & Seismic monitoring & 2000L, 4100L & {$[31],[32],[33]$} \\
\hline \multicolumn{4}{|l|}{ Biology } \\
\hline Biodiversity (BHSU/SDSMT) & Microbiology & $\begin{array}{l}\text { Surface, 300L, 2000L, } \\
\text { 4100L, 4550L, 4850L }\end{array}$ & [34] \\
\hline Biofuels (SDSMT) & Biofuels & $4550 \mathrm{~L}, \mathbf{4 8 5 0 L}, 5000 \mathrm{~L}$ & $\begin{array}{l}{[35],[36],[37],[38],[39],} \\
{[40],[41],[42]}\end{array}$ \\
\hline Bioprocessing R\&D (SDSMT) & Biofuels & $4850 \mathrm{~L}$ & \\
\hline Syngas/Biofuels (SDSMT) & Biofuels & $4850 \mathrm{~L}$ & [43], [44], [45] \\
\hline $\begin{array}{l}\text { Life Underground - } \\
\text { NASA Astrobiology Institute }\end{array}$ & Water in drill holes, geomicrobiology & $\begin{array}{l}\text { Surface, 800L, } \\
\text { 1700L, 2000L, 4850L }\end{array}$ & \\
\hline Engineering ${ }^{\dagger}$ & & & \\
\hline
\end{tabular}

${ }^{*}$ Publication in progress.

$\dagger$ No active engineering research currently, but there are interested groups. Previous groups investigated electromagnetic signal propagation in rock tunnels and autonomous submersibles. 


\section{FUTURE SCIENCE}

The are many promising options to expand the number of projects both in the near-term and into the next decade. The locations of existing and future experiments is shown in Figure 9.

\section{Low-Background Counting Facility}

Several proposals have been submitted proposing to develop state-of-the-art low background counting detectors and facilities at the 4850L at SURF making use of existing infrastructure and/or excavations. One possible location for a low-background counting facility is in the SE shop space at the 4850L Ross Campus.

\section{CASPAR/DIANA}

As part of the NSF support for the development of underground physics experiments, a proposal has been developed to create an underground accelerator facility for low-energy nuclear astrophysics experiments. The DIANA project (Dual Ion Accelerators for Nuclear Astrophysics) would consist of two high-current accelerators offering a low-energy beam (30-400 kV) and a high-energy beam (350-3000 kV) [46].

Planning has begun for the first phase of the project known as the Compact Accelerator System for Performing Astrophysical Research (CASPAR). It will consist of a 1-MV Van de Graff accelerator that will be re-located from the University of Notre Dame. Installation in the NE shop of the 4850L Ross Campus could begin by the end of 2014 with commissioning in 2015 .

\section{Next Generation Dark Matter}

The DOE and NSF have plans to develop Generation-2 dark matter experiments, with masses up to $\sim 10$ tonnes. The LUX-ZEPLIN (LZ) collaboration has submitted a proposal to develop a G-2 experiment in SURF using the existing space and infrastructure in the Davis Campus. Construction could begin as early as 2016 followed by commissioning in 2017. SURF is open to working with all interested collaborations to develop proposals for G-2 experiments.

The DOE has discussed developing a "roadmap" for dark matter searches including Generation-2 and Generation-3 dark matter experiments beginning FY17. Initial efforts to develop the Generation-3 collaboration are advancing.

\section{Neutrinoless Double-Beta Decay}

The MAJORANA collaboration is investigating whether it would be possible to perform the next-generation neutrinoless double-beta decay experiment on the 4850L at SURF. If the combination of rock overburden and veto was determined to be sufficient, a new laboratory may be needed.

\section{Long Baseline Neutrino Experiment (LBNE)}

The LBNE experiment offers a compelling science program that will enhance the our understanding of the neutrino in several fundamental ways [47]. A large liquid argon detector (or detectors) would observe neutrinos generated at Fermilab using an upgraded accelerator beam (up to $2.3 \mathrm{MW}$ ). The detector mass could be staged (eg., $10 \mathrm{kt}, 17 \mathrm{kt}$, $34 \mathrm{kt}$, etc).

Designs have been developed for an initial detector deployment on the surface, but the new planning baseline focuses on the installation underground on the 4850L. The reduced backgrounds due to cosmic ray muons [48] significantly enhances the physics reach of the LBNE detector. Construction may start late 2017 with transition to operations by 2023. 


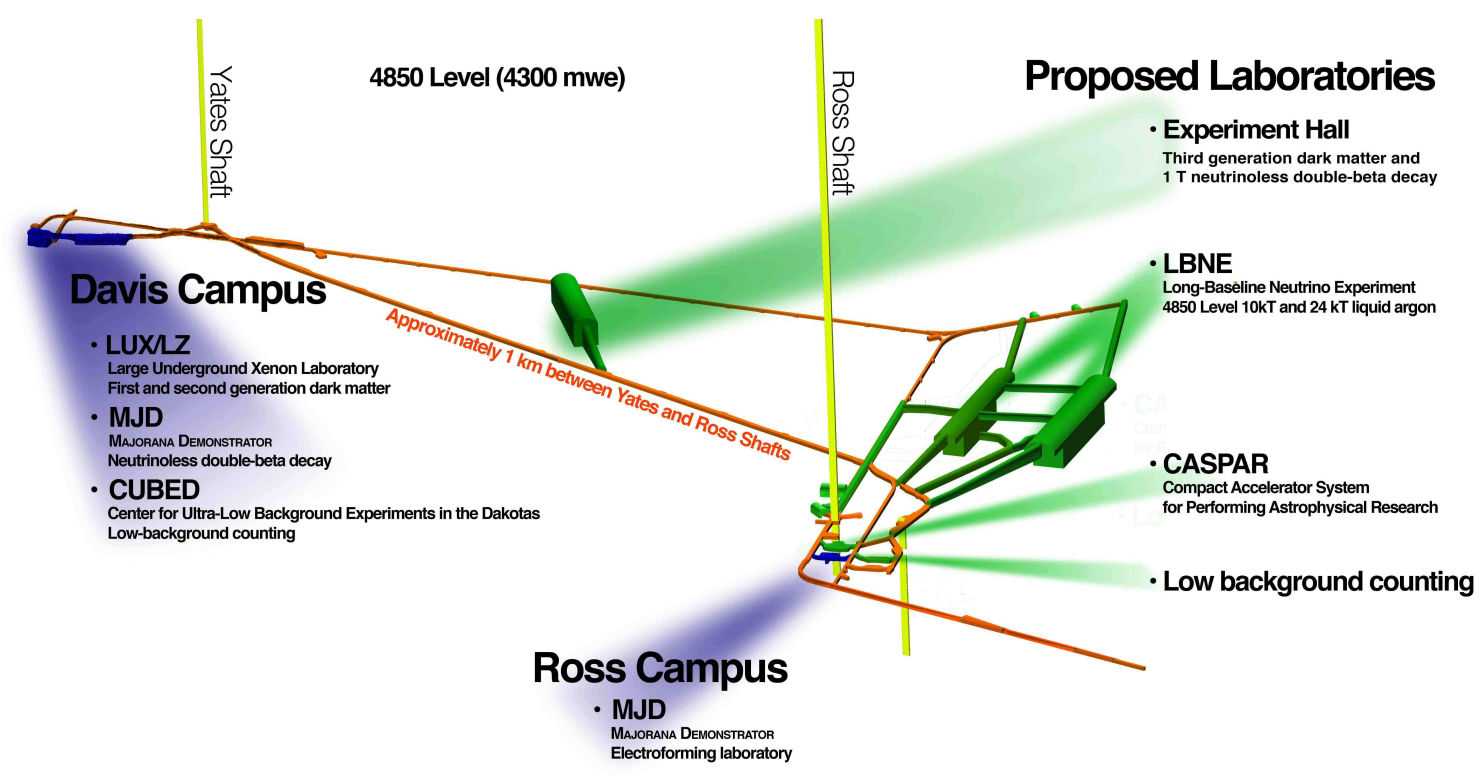

FIGURE 9. The 4850 level of SURF highlighting the existing and proposed experiments.

\section{SUMMARY}

SURF is a deep underground research facility, dedicated to scientific uses. Research activities are supported at a number of facilities, both on the surface and underground. Two campuses on the $4850 \mathrm{~L}$ accommodate a number of leading efforts, and in particular the 4850L Davis Campus has been successfully operating for 18 months. The LUX and MAJORANA experiments are well established and there will soon be capabilities for low-background counting. Many expansion possibilities are on the horizon and a number of key experiments in the U.S. research program are developing plans for installation at SURF.

\section{REFERENCES}

1. K. T. Lesko, Eur. Phys. J. Plus 127, 107 (2012).

2. J. Heise, Nuc. Phys. A 834, 805c-808c (2010).

3. S. T. Mitchell, Nuggets to Neutrinos: The Homestake Story, Xlibris, 2009.

4. K. T. Lesko, Why the US needs a deep domestic research facility: Owning rather than renting the education benefits, technology advances, and scientific leadership of underground physics (2013), arXiv:1304.0402 [physics.ins-det].

5. K. T. Lesko, et al., Deep Underground Science and Engineering Laboratory — preliminary design report (2012), arXiv: 1108.0959 [hep-ex].

6. L. C. Murdoch, L. N. Germanovich, H. Wang, T. C. Onstott, D. Elsworth, L. Stetler, and D. Boutt, Hydrogeol. J. 20, $27-43$ (2012).

7. G. Zhan, and T. Duex, Mining Eng. 64(4), 64-68 (2010).

8. W. G. Pariseau, D. R. Tesarik, and T. C. Trancynger, Transactions of the Society for Mining, Metallurgy, and Exploration 332, 370-388 (2012).

9. S. W. Caddey, R. L. Bachman, T. J. Campbell, R. R. Reid, and R. P. Otto, U.S. Geol. Surv. Bull. 1857-J, 67 (1991).

10. W. Roggenthen, and A. R. Smith (2008).

11. A. R. Smith (2007 and updates through 2011).

12. Y.-D. Chan (2012), http://www. sanfordlab.org/lbnl/186.

13. K. J. Thomas, Radon monitoring and the role of iron oxide on radon emanation at the Sanford Laboratory at Homestake in Lead, South Dakota, Master's thesis (unpublished), University of South Dakota, Department of Physics (2011).

14. D.-M. Mei, C. Zhang, K. Thomas, and F. Gray, Astropart. Phys. 34, 33-39 (2010), arXiv: 0912.0211 [nucl-ex] .

15. F. E. Gray, C. Ruybal, J. Totushek, D.-M. Mei, K. Thomas, and C. Zhang, Nucl. Instrum. Meth. A 638, 63-66 (2010), arXiv: $1007.1921[$ nucl-ex].

16. B. T. Cleveland, T. Daily, J. R. Davis, J. R. Distel, K. Lande, C. K. Lee, P. S. Wildenhain, and J. Ullman, Astrophys. J. 496, 505-526 (1998). 
17. D. S. Akerib, et al. (2013), arXiv:1310.8214 [astro-ph.CO].

18. N. Abgrall, et al. (2013), arXiv:1308.1633 [physics.ins-det].

19. B. Szczerbinska, et al., Proceedings of the South Dakota Academy of Science 89, 23 (2010).

20. D. Tiedt, Radioactive Background Simulation and Cleanliness Standards Analysis for the Long Baseline Neutrino Experiment Located at the Sanford Underground Research Facility, Master's thesis (unpublished), South Dakota School of Mines and Technology, Department of Physics (2013).

21. J. Harms, et al., Class. Quant. Grav. 27, 225011 (2010), arXiv:1006.0678 [ gr-qc] .

22. F. Acernese, R. De Rosa, R. De Salvo, F. Garufi, G. Giordano, J. Harms, V. Mandic, A. Sajeva, T. Trancynger, and F. Barone, J. Phys.: Conf. Ser 228, 012036 (2010).

23. J. R. Gage, D. Fratta, A. L. Turner, M. M. MacLaughlin, and H. F. Wang, Int. J. Rock Mech. Mining Sci. 61, 244-255 (2013).

24. J. Volk, et al., J. Inst. 7, P01004 (2012), arXiv: 1205.1777 [physics . acc-ph] .

25. J. Volk, V. Shiltsev, A. Chupyra, M. Kondaurov, S. Singatulin, D. Fratta, A. Meulemans, C. Potier, and H. Wang, 12th International Workshops on Accelerator Alignment IWAA, 10-14 September, Fermilab (2012).

26. H. F. Wang, J. R. Gage, D. O. Fratta, M. M. MacLaughlin, and A. Turner, Proceedings of the 8th International Workshop on Structural Health Monitoring, 13-15 September, Stanford, CA p. 8 pp (2011).

27. J. R. Gage, N. Noni, A. Turner, M. MacLaughlin, and H. F. Wang, 44th U.S. Rock Mechanics Symposium and 5th U.S-Canada Rock Mechanics Symposium, 27-30 June, Salt Lake City, UT p. 8 pp (2010).

28. J. Kennedy, L. Murdoch, A. J. Long, and K. Koth, American Geophysical Union Fall Meeting, 5-9 December, San Francisco, CA Abstract NH54A-01, A1 (2011).

29. A. E. Armstrong, Hydrothermal alteration and gold mineralization in biotite zone host rocks at Homestake Mine in Lead, South Dakota, Master's thesis (unpublished), South Dakota School of Mines and Technology, Department of Geology (2013).

30. R. C. Hamer, Hydrothermal alteration and gold deposition in the Homestake iron-formation-hosted gold deposit, Lead, South Dakota, Master's thesis (unpublished), South Dakota School of Mines and Technology, Department of Geology (2010).

31. W. M. Roggenthen, and C. D. Koch, Proc. 47th U.S. Rock Mech./Geomech. Symp. 23-26 June, 2013, San Francisco, CA Paper 13-493, 8 (2013).

32. C. S. Sherman, M. Magliocco, and S. D. Glaser, 45th U.S. Rock Mech./Geomech. Symp., 26-29 June, San Francisco, CA Paper 11-526, 7 (2011).

33. J. K. Van Beek, W. M. Roggenthen, M. Magliocco, and S. D. Glaser, Proceedings of the 3rd CANUS Rock Mechanics Symposium, May 2009, Toronto, ON Paper 4155, 7 (2009).

34. E. J. Waddell, T. J. Elliott, J. M. Vahrenkamp, W. M. Roggenthen, R. K. Sani, C. M. Anderson, and S. S. Bang, Env. Tech. 31:8-9, 979-991 (2010).

35. A. Bhalla, A. Kainth, and R. K. Sani, Genome Announc. 1, e00595-13 (2013).

36. G. Rastogi, R. N. Gurram, A. Bhalla, R. Jaswal, R. Gonzalez, K. M. Bischoff, S. R. Hughes, K. Sudhir, and R. K. Sani, Journal of Frontiers in Microbiotechnology, Ecotoxicology and Bioremediation 4, 1-8 (2013).

37. A. Bhalla, N. Bansal, S. Kumar, and R. K. Sani, Bio. Tech. 128, 751-759 (2013).

38. G. Rastogi, R. N. Gurram, A. Bhalla, R. Gonzalez, K. M. Bischoff, S. R. Hughes, S. Kumar, and R. K. Sani, Front. Microbiol. 4, 18 (2013).

39. G. Rastogi, A. Bhalla, A. Adhikari, K. M. Bischoff, S. R. Hughes, L. P. Christopher, and R. K. Sani, Bio. Tech. 101, 8798-8806 (2010).

40. G. Rastogi, S. Osman, R. K. Kukkadapu, M. Engelhard, P. A. Vaishampayan, G. L. Andersen, and R. K. Sani, Microb. Ecol. 60, 539-550 (2010).

41. G. Rastogi, G. L. Muppidi, R. N. Gurram, A. Adhikari, K. M. Bischoff, S. R. Hughes, W. A. Apel, S. S. Bang, D. J. Dixon, and R. K. Sani, J. Ind. Microbiol. Biotechnol. 36, 585-598 (2009).

42. G. Rastogi, L. D. Stetler, B. M. Peyton, and R. K. Sani, J. Microb. 47, 371-384 (2009).

43. A. Shende, D. Harder-Heinze, and R. Shende, Proceedings of the South Dakota Academy of Science 91, 208 (2012).

44. A. Shende, R. Jaswal, D. Harder-Heinze, A. Menan, and R. Shende, Technical Proceedings of the 2012 NSTI Nanotechnology Conference and Expo 3, 489 (2012).

45. A. Shende, R. Jaswal, and R. Shende, Proceedings of AIChE Annual Meeting ISBN: 978-1-4665-6277-6, 120 (2012).

46. A. Best, M. Couder, M. Famiano, A. Lemut, and M. Wiescher, Nucl. Instrum. Meth. A 727, 104-108 (2013), http://www.jinaweb.org/underground/DIANA.

47. C. Adams, et al., Scientific opportunities with the Long-Baseline Neutrino Experiment (2013), arXiv:1307. 7335 [hep-ex].

48. D. Barker, D.-M. Mei, and C. Zhang, Phys. Rev. D 86, 054001 (2012), arXiv: 1202.5000 [physics.ins-det] . 